BULL. AUSTRAL. MATH. SOC.

VOL. $31(1985), 349-354$.

\title{
INDECOMPOSABLE 2-GROUPS WITH ALL AUTOMORPHISMS CENTRAL
}

\author{
M.J. CURRaN
}

\begin{abstract}
This paper provides examples of indecomposable 2-groups with non-abelian automorphism groups in which every automorphism is central.
\end{abstract}

The papers by Malone [4] and the author [1] give examples of nonabelian $p$-groups with non-abelian automorphism groups in which every automorphism is central, and provide background material on the significance of these results. The groups given in these examples, however, all have an abelian direct factor and Malone questions in [4] whether there are indecomposable $p$-groups with the same property. When $p=2$, the groups numbered 91,92 and 99 in the Hall and Senior tables [3] are indecomposable and the only groups in the tables with all their automorphisms central. But in each case the full automorphism group is abelian (see [1]). However, this paper shows that each of these groups can be generalized to give an indecomposable 2-group with non-abelian automorphism group in which every automorphism is central. The notation used is standard and that of Gorenstein [2].

Consider the following groups:

Received 2 November 1984. The author is indebted to Professor R.R. Struik for drawing his attention to the possibility of generalizing groups 91 and 92 in this way.

Copyright Clearance Centre, Inc. Serial-fee code: 0004-9727/85 $\$ \mathrm{~A} 2.00+0.00$. 


$$
\begin{aligned}
& G_{1}=\langle a, b, c, d| a^{2^{m}}=b^{2^{m}}=c^{2}=d^{2}=1,[a, c]=d,[b, c]=a^{2^{m-1}}, \\
& [c, d]=1,\langle a, b, d\rangle \text { abelian, } m \geq 2\rangle \text {; } \\
& G_{2}=\langle a, b, c| a^{2^{n}}=b^{2^{n}}=c^{2^{n}}=1,[a, b]=c^{2^{n-1}},[b, c]=b^{2^{n-1}}, \\
& [a, c]=1, n \geq 2\rangle \text {; }
\end{aligned}
$$

$$
\begin{array}{r}
G_{3}=\langle a, b, c| a^{2^{r}}=b^{4}=c^{2^{s}}=1,[a, b]=a^{2^{r-1}},[b, c]=b^{2}, \\
[a, c]=1, r>s, r \geq 3, s \geq 1\rangle .
\end{array}
$$

When $m=2, n=2, r=3$ and $s=1$, the groups $G_{1}, G_{2}, G_{3}$ become the groups 91, 92, 99 respectively of the Hall and Senior tables, and as noted above these groups have abelian automorphism groups. When $s=1, G_{3}$ becomes the group discussed by Struik [5], and this group, as the main result of [5] shows, has an abelian automorphism group. Thus, the proposition below, besides giving the groups promised in the abstract, also provides a unified proof that these automorphism groups are abelian.

We begin with a lemma, containing some obvious facts about $G_{1}, G_{2}$ and $G_{3}$.

LEMMA 1. (i) $z\left(G_{1}\right)=\left\langle a^{2}, b^{2}, d\right\rangle ; z\left(G_{2}\right)=\left\langle a^{2}, b^{2}, c^{2}\right\rangle=z\left(G_{3}\right)$.

(ii) For $1 \leq i \leq 3$, each $G_{i}$ has an abelian subgroup $A_{i}$ of index 2 , where $A_{1}=\langle a, b, d\rangle ; A_{2}=\left\langle a, b^{2}, c\right\rangle=A_{3}$.

(iii) $\quad G_{1}^{\prime}=\left\langle a^{2^{m-1}}, d\right\rangle ; G_{2}^{\prime}=\left\langle b^{2^{n-1}}, c^{2^{n-1}}\right\rangle ; G_{3}^{\prime}=\left\langle a^{2^{2-1}}, b^{2}\right\rangle$.

Now writing $G$ for $G_{i}$ and $A$ for $A_{i}(1 \leq i \leq 3)$ we summarize some properties of $G$.

LEMMA 2. (i) $Z(G)=\Phi(G)$.

(ii) $G^{\prime} \leq Z(G)$.

(iii) $A$ is a characteristic subgroup of $G$.

Proof. (i) follows since $G / G^{\prime}$ has rank 3 and $G / Z(G)$ is an 
elementary abelian group of order 8 .

(ii) is obvious.

(iii) $A$ is characteristic since it is the only abelian subgroup of index 2 in $G$. For if $B$ were another such subgroup, then $G=A B$ implies $|G: A|=|B: A \cap B|$ and so $|G: A \cap B|=4$. But $A \cap B \leq Z(G)$ and yet $|G: Z(G)|=8$, a contradiction.

PROPOSITION. $G=G_{i}(1 \leq i \leq 3)$ is an indeciomposable 2-group with all automorphisms central. Further, the automorphism groups of $G_{1}, G_{2}, G_{3}$ are abelian when $m=2, n=2, s=1$ respectively, and non-abelian otherwise.

Proof. $G=(a, b, c)$ cannot be the direct product of two non-abelian groups. But if $G$ has an abelian direct factor $H$ say, then $H \leq Z(G)=\Phi(G)$, so $H$ is trivial. Thus $G$ is indecomposable.

Let $\theta$ be an arbitrary automorphism of $G$ which maps the generators $a, b, c$ of $G$ onto $\bar{a}, \bar{b}, \bar{c}$ respectively. We show each of the elements $a^{-1} \bar{a}, b^{-1} \bar{b}, c^{-1} \bar{c}$ lies in the centre of $G$. Thus $g^{-1} g^{\theta} \in Z(G)$ for any $g \in G$, and so $\theta$ is a central automorphism.

Firstly, note every element in $G$ can be written uniquely in the form $a^{i} b^{j} c^{k} z$, for some integers $i, j, k$ with $0 \leq i, j, k \leq 1$ and some $z \in Z(G)$. Since $Z(G)$ is characteristic and $a, b, c \in G \backslash Z(G)$, each of $\bar{a}, \bar{b}, \bar{c}$ is of the form $a^{i} b^{j} c^{k} z$, where $(i, j, k) \neq(0,0,0)$. We now examine the 3 cases. For convenience in this discussion $i, j, k, l, t, u$ are all integers with $0 \leq i, j, k, \tau, t, u \leq 1$, and $z_{a}, z_{b}, z_{c} \in Z(G)$.

(1) $G=G_{1}$. Since $a, b \in A$, a characteristic subgroup, $\bar{a}=a^{i} b^{j} z_{a}, \bar{b}=a^{k} b^{2} z_{b}$, where $(i, j) \neq(0,0),(k, i) \neq(0,0)$. However $\mho^{m-1}(A) \cap G^{\prime}=\left\langle a^{2^{m-1}}\right\rangle$ is characteristic in $G$, so $j=0$ and $\imath=1$. That is, $\bar{a}=a z_{a}, \bar{b}=a^{k} b z_{b}$. Now $c \notin A$ so $\bar{c}=a^{t} u_{c z_{c}}$. But $c$ has order 2 , so $t=u=0$ and $z_{c}^{2}=1$. That is, $\vec{c}=c z_{c}$. Finally, from a defining relation for $G$ and using the fact that 
$G^{\prime} \leq Z(G):$

$$
a^{2^{m-1}}=\bar{a}^{2^{m-1}}=[\bar{b}, \bar{c}]=\left[a^{k} b, c\right]=\left[a^{k}, c\right][b, c]=d^{k} a^{2^{m-1}} \text {, so } k=0 .
$$

Thus $\bar{a}=a z_{a}, \bar{b}=b z_{b}, \bar{c}=c z_{c}$, where $z_{c}^{2}=1$.

(2) $G=G_{2}$. As in (1) since $a, c \in A$ and $\mho^{n-1}(A) \cap G^{\prime}=\left\langle c^{2^{n-1}}\right\rangle$, $\bar{a}=a c^{j} z_{a}, \bar{c}=c z_{c}$. Now $b \notin A$, so $\bar{b}=a^{t} b c^{u} z_{b}$. But $G^{\prime}$ is characteristic so $t=0$. That is, $\bar{b}=b c^{u_{z}} z_{b}$. Finally, from the defining relations for $G$ and using the fact that $G^{\prime} \leq Z(G)$ :

$$
\begin{aligned}
b^{2^{n-1}}\left(c^{2^{n-1}}\right)^{u} & =b^{2^{n-1}}=[\bar{b}, \bar{c}]=\left[b c^{u}, c\right]=[b, c]=b^{2^{n-1}}, \text { so } u=0 ; \\
c^{2^{n-1}} & =\bar{c}^{2^{n-1}}=[\bar{a}, \bar{b}]=\left[a c^{j}, b\right]=[a, b]\left[c^{j}, b\right]=c^{2^{n-1}}\left(b^{2^{n-1}}\right)^{j}, \\
& \text { so } j=0 .
\end{aligned}
$$

Thus $\bar{a}=a z_{a}, \quad \bar{b}=b z_{b}, \bar{c}=c z_{c}$.

(3) $G=G_{3}$. As in (1) since $a, c \in A, \bar{a}=a^{i} c^{j} z_{a}, \bar{c}=a_{c}^{k} z_{c}$, where $(i, j) \neq(0,0),(k, z) \neq(0,0)$. However, a has order $2^{r}$ and $c$ has order $2^{s}$ where $r>s$, so $i=1, k=0$ and $z_{c}^{2^{s}}=1$. That is $\bar{a}=a c z_{a}, \bar{c}=c z_{c}$. Now $b \notin A$ so $\bar{b}=a^{t} b c^{u} z_{b}$. However $b$ has order 4 so $t=0$. That is $\bar{b}=b c^{u_{z}} z_{b}$. Finally, from the defining relations for $G$ and using the fact that $G^{\prime} \leq Z(G)$ :

$$
\begin{aligned}
& a^{2^{r-1}}=\bar{a}^{2^{r-1}}=[\bar{a}, \bar{b}]=\left[a c^{j}, b c^{u}\right]=[a, b]\left[c^{j}, b\right]=a^{2^{r-1}} b^{2^{j}}, \\
& b^{2}=[b, c]=\left[b c^{u}, c\right]=[\bar{b}, \bar{c}]=b^{2}= \begin{cases}b^{2} z_{b}^{2} & \text { if } u=0, \\
& \\
c^{2} z_{b}^{2} & \text { if } u=1,\end{cases}
\end{aligned}
$$


so $u=0$ and $z_{b}^{2}=1$. Thus $\bar{a}=a z_{a}, \bar{b}=b z_{b}, \bar{c}=c z_{c}$ where $z_{b}^{2}=1, z_{c}^{2^{s}}=1$

Thus in each case all automorfhisms are central. Further, each of the possibilities listed above for $\bar{a}, \bar{b}, \bar{c}$ occur as the image of an automorphism of $G$. For $\bar{a}, \bar{b}, \bar{c}$ satisfy the same relations as $a, b, c$ and $G=\langle a, b, c\rangle=\left\langle a z_{a}, b z_{b}, c z_{c}, z_{a}, z_{b}, z_{c}\right\rangle=\langle\bar{a}, \bar{b}, \bar{c}\rangle$, since $Z(G) \leq \Phi(G)$. In particular, suppose $m>2$ in $G_{1}, n>2$ in $G_{2}$ and $s>1$ in $G_{3}$. Then let $\theta_{i}(1 \leq i \leq 3)$ be the automorphisms which map $a$ to $a b^{2}$ in $G_{1}, b$ to $b^{-1}$ in $G_{2}, a$ to $a c^{2}$ in $G_{3}$ respectively, and let $\psi_{i}(1 \leq i \leq 3)$ be the automorphisms which map $b$ to $b a^{2}$ in $G_{1}, c$ to $c b^{2}$ in $G_{2}, c$ to $c a^{2^{r-s}}$ in $G_{3}$ respectively (where all other generators are fixed by these automorphisms). Now an easy calculation shows $\theta_{i} \psi_{i} \neq \psi_{i} \theta_{i}$, for $1 \leq i \leq 3$, so Aut $G_{i}$ is non-abelian.

Finally, let $m=2$ in $G_{1}$, let $n=2$ in $G_{2}$ and let $\theta$ be an arbitrary (central) automorphism of $G=G_{i} \quad(1 \leq i \leq 2)$ as listed above. Then $a^{2}, b^{2}, c^{2}$ are fixed by $\theta$ since $Z(G)$ has exponent 2 , and $d \in G_{1}^{\prime}$ is also fixed. Thus $\theta$ fixes $Z(G)$ and so $\theta^{2}=1$, that is Aut $G$ is (elementary) abelian. If $s=1$ in $G_{3}$, then any (central) automorphism $\theta$ of $G_{3}$ as listed above is of form $a^{\theta}=a^{2 j+1} g_{a}$ $\left(0 \leq j \leq 2^{r-1}-1\right), b^{\theta}=b g_{b}, c^{\theta}=c g_{c}$, where $g_{a}, g_{b}, g_{c} \in G_{3}^{\prime}$ and are fixed by any automorphism of $G_{3}$. Clearly any two automorphisms of this form commute so Aut $G$ is abelian. 


\section{References}

[1] M.J. Curran, "A non-abelian automorphism group with all automorphisms central", BulZ. Austral. Math. Soc. 26 (1982), 393-397.

[2] Daniel Gorenstein, Finite groups (Harper and Row, New York, Evanston and London, 1968).

[3] Marshall Hall, Jr. and James K. Senior, The groups of order $2^{n}$ $(n \leq 6)$ (Macmillan, New York; Collier-Macmillan, London, 1964).

[4] J.J. Malone, "p-groups with non-abelian automorphism groups and all automorphisms central", BulZ. Austral. Math. Soc. 29 (1984), $35-37$.

[5] Ruth Rebekka Struik, "Some non-abelian 2-groups with abelian automorphism groups", Arch. Math. (Basel) 39 (1982), 299-302.

Department of Mathematics and Statistics,

University of Otago,

Box 56,

Dunedin,

New Zealand. 